\title{
Monoenergetic electron bunches generated from thin solid foils irradiated by ultrashort, ultraintense circularly polarized lasers
}

\author{
Wei-Min Wang, ${ }^{1,3}$ Zheng-Ming Sheng, ${ }^{1,2}, *$ Yu-Tong Li, ${ }^{1}$ Li-Ming Chen, ${ }^{1}$ Shigeo Kawata, ${ }^{3}$ and Jie Zhang ${ }^{1,2}$ \\ ${ }^{1}$ Beijing National Laboratory of Condensed Matter Physics, Institute of Physics, CAS, Beijing 100190, China \\ ${ }^{2}$ Department of Physics, Shanghai Jiao Tong University, Shanghai 200240, China \\ ${ }^{3}$ Graduate School of Engineering, Utsunomiya University, 7-1-2 Yohtoh, Utsunomiya 321-8585, Japan
}

(Received 5 February 2010; published 2 July 2010)

\begin{abstract}
Future ultrashort $10^{22}-10^{25} \mathrm{~W} \mathrm{~cm}^{-2}$ laser pulses offer the possibility of producing ultrashort monoenergetic electron beams in the $\mathrm{GeV}-\mathrm{TeV}$ level by direct laser ponderomotive force acceleration (LPFA) in distances of millimeters to about $1 \mathrm{~m}$. A scheme is proposed that a thin solid foil and a thick solid foil are placed on the laser axis, where the thin foil supplies the electron source for LPFA and the thick foil reflects the laser away, however allows the accelerated electrons to go through. By optimizing the distance between the foils, one can obtain the maximum electron beam energy. This scheme is demonstrated by particle-in-cell simulations.
\end{abstract}

DOI: 10.1103/PhysRevSTAB.13.071301

PACS numbers: 52.38.Kd, 41.75.Lx, 52.65.Rr

\section{INTRODUCTION}

High energy monoenergetic electron beams produced by laser-plasma-based acceleration are promising for broad applications such as radiotherapy in medicine [1,2], radiography in biology and material science [3], terahertz radiation generation [4,5], and electron-positron colliding physics, etc. With the development of ultrashort high power laser technology, significant progress in the laserplasma-based acceleration has been made in the past decade. Especially, the laser wakefield acceleration (LWFA) in both the linear regime and the highly broken wave regime, i.e., the bubble regime or blowout regime, has been studied extensively [6-16]. With the advent of the lasers with the intensity higher than $10^{22} \mathrm{~W} \mathrm{~cm}^{-2}$ and the duration of a few fs, e.g., the extreme light infrastructure (ELI) to be constructed in the near future, the laser-plasmabased acceleration presents new opportunities as well as challenges. For example, in LWFA, to optimize the wakefield generation, the plasma wavelength should be about the laser duration, which requires the plasma electron density $n_{e 0}$ approaching the critical density $n_{c}$ $\left(10^{21} \mathrm{~cm}^{-3}\right)$ for a laser with the duration a few of fs. This limits the phase velocity of the wakefield and, consequently, the final energy of the electron beam. In the bubble or blowout regime, the electron energy can reach $0.34\left(a_{0} n_{c} / n_{e 0}\right) \mathrm{MeV} \sim 0.34 a_{0} \mathrm{MeV}$ according to the scaling given in Ref. [12], where $a_{0}$ is the laser amplitude normalized by $m_{e} \omega_{0} c / e=32.1 \mathrm{GV} / \mathrm{cm}$ for $\lambda_{0}=$ $2 \pi c / \omega_{0}=1 \mu \mathrm{m}, c$ is the speed of light in the vacuum, and $e$ and $m_{e}$ are the electron charge and rest mass, respectively. Then the laser with the intensity of

*zmsheng@sjtu.edu.cn
$10^{22} \mathrm{~W} \mathrm{~cm}^{-2}$ can produce only about $30 \mathrm{MeV}$ electron beams. Obviously, such laser parameters are not optimized for LWFA. On the other hand, such a laser presents a good opportunity for laser ponderomotive force acceleration (LPFA). In terms of the analysis below, the ponderomotive force $\sim \pi a_{0}^{2} / 2 \tau_{0}$ is proportional to the laser intensity and the reciprocal of the laser duration $\tau_{0}$. Thus ultrashort ultraintense lasers are able to drive LPFA very efficiently and electron energy up to $0.255 a_{0}^{2} \mathrm{MeV}$ can be obtained, which can exceed that in LWFA. For instance, when the laser intensity is $10^{22} \mathrm{~W} \mathrm{~cm}^{-2}$ and the duration is four laser periods or $13.3 \mathrm{fs}$, the ponderomotive force is up to $46 \mathrm{TV} / \mathrm{cm}$ and electron energy can reach about $1 \mathrm{GeV}$. The corresponding acceleration distance of electrons is $l_{\text {acc }}=\tau_{0} a_{0}^{2} / 8=1.8 \mathrm{~mm}$, much larger than the laser duration. Therefore, the electron beam can be separated from the laser easily before it is decelerated.

How can one inject electrons into the acceleration phase of the ponderomotive force? How can one control the energy spread of electron beams to a small value? In this paper, we propose to use a nanothickness foil irradiated by a circularly polarized (CP) laser for the generation of high quality electron beams. For a thin enough foil, the electrostatic (ES) field formed by the charge separation can be overtaken easily by the laser ponderomotive force (or equivalently the "ponderomotive field" hereafter given by the ponderomotive force divided by the charge), i.e., the foil electrons can be governed and trapped by the ponderomotive field easily ("trapped" here means that the electrons stay in the acceleration phase of the ponderomotive field). For example, the maximum ES field for the foil with the thickness $1 \mathrm{~nm}$ and the density $100 n_{c}$ is $125 \mathrm{GV} / \mathrm{cm}$, which is equal to the ponderomotive field of the laser with the intensity $3 \times 10^{19} \mathrm{~W} \mathrm{~cm}^{-2}$ and the 


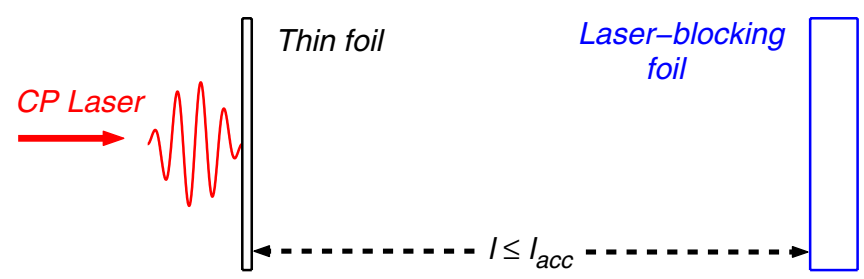

FIG. 1. (Color) Schematic plot for laser ponderomotive force acceleration, where a thin solid foil (source foil) provides the electron source for laser acceleration and a thick solid foil (laserblocking foil) blocks the laser when the electrons reach the maximum energy.

duration 13.3 fs. Besides, the trapped electron beam will be very short and thus its energy spread will be very small because all the electrons experience nearly the same ponderomotive force, if a thin foil is used. Here, we use a CP laser pulse rather than a linearly polarized laser pulse because a CP pulse has a larger acceleration phase of the ponderomotive field in the whole rising front of the pulse. Electrons can stay (or be trapped) in the acceleration phase of the ponderomotive field for a long time and be accelerated efficiently.

According to what is mentioned above, a scheme can be proposed that a thin source foil and a thick laser-blocking foil are placed on the laser propagation axis and their distance is smaller than $l_{\text {acc }}$, as shown in Fig. 1. The electrons of the thin source foil are pushed away from the ions by a CP laser pulse and tapped by the ponderomotive field. When the trapped electrons are accelerated to the maximum energy in a distance of $l_{\text {acc }}$, the laser meets the laser-blocking foil and is reflected away. However, the electrons pass through it. Then a high energy electron beam is obtained. The distance between the two foils can also be adjusted to control the electron beam energy. In this scheme, electrons are injected from the front of the laser. Electrons located initially at the relative back positions in the foil will meet the laser front first and are accelerated at the earlier time. They have larger velocities than those initially at the deeper (or relative front) positions. During the accelerating process, the distance between them decreases gradually. Then the energy spread is reduced since the electron energy depends on their positions in the laser pulse, as shown in Eq. (2) in Sec. II. It should be noted that another method [17] was proposed to accelerate electrons by use of the laser ponderomotive force, too. However, it demands a tenuous plasma to slow down the laser pulse and preacceleration of electrons before they are accelerated by the ponderomotive force. The detailed discussion can be found in Ref. [17] and the references cited in it.

In the following, we predict the electron energy and acceleration distance by the single electron model $[18,19]$. Then these predictions and the scheme proposed above are examined and demonstrated by one-dimensional (1D) and two-dimensional (2D) particle-in-cell (PIC) simulations.

\section{SINGLE ELECTRON MODEL}

The motion of a test electron in a laser field is governed by the Hamiltonian $H=\gamma-\phi$, where $\gamma$ is the electron relativistic factor and $\phi$ is the scalar potential normalized by $m_{e} c^{2} / e$. Assume that the laser propagates along the $+x$ direction. In the $1 \mathrm{D}$ case, the electron transverse momenta are expressed by $p_{y}=A_{y}$ and $p_{z}=A_{z}$. Here the electron momenta are normalized by $m_{e} c$ as well as $A_{y}$ and $A_{z}$ are the vector potentials normalized by $m_{e} c^{2} / e$. Taking the partial derivative of $H$ with respect to $x$, one obtains $d p_{x} / d t=-\partial(\gamma-\phi) / \partial x$, where the first term is the ponderomotive force and the second is the ES force. Assume that the laser waveform does not vary with the time in its propagation. Then $A_{y}$ and $A_{z}$ are the functions of $\xi=t-$ $x$, where $t$ and $x$ are normalized by the laser period $T_{0}$ and wavelength $\lambda_{0}$. One can obtain a conversed quantity $H-$ $p_{x}$, which gives $\gamma-\phi-p_{x}=1$. Here, we have assumed that the electron momenta vanish initially as well as scalar and vector potentials. When the ES field is always much smaller than the ponderomotive field, i.e. $|\partial \phi / \partial x| \ll$ $|\partial \gamma / \partial x|$, one can consider $\phi \simeq 0$ and obtain $\gamma-p_{x}=1$. Then $p_{x}=\left(A_{y}^{2}+A_{z}^{2}\right) / 2$ and $\gamma=\left(A_{y}^{2}+A_{z}^{2}\right) / 2+1$ are derived [18].

Consider the $\mathrm{CP}$ laser field with the following form:

$A_{y}=a_{0} \sin \left(\pi \xi / \tau_{0}\right) \sin (2 \pi \xi), \quad 0 \leq \xi<\tau_{0}$, $A_{z}=a_{0} \sin \left(\pi \xi / \tau_{0}\right) \cos (2 \pi \xi), \quad 0 \leq \xi<\tau_{0}$.

Inserting Eq. (1) into the expressions of electron momenta, one obtains

$$
p_{x}=\frac{a_{0}^{2}}{2} \sin ^{2}\left(\pi \xi / \tau_{0}\right), \quad \gamma=\frac{a_{0}^{2}}{2} \sin ^{2}\left(\pi \xi / \tau_{0}\right)+1 .
$$

In terms of Eq. (2), the ponderomotive field is $-\partial \gamma / \partial x=$ $\left[\left(\pi a_{0}^{2}\right) /\left(2 \tau_{0}\right)\right] \sin \left(\frac{2 \pi \xi}{\tau_{0}}\right)$. When $\xi<\tau_{0} / 2$, the electron is accelerated. Until $\xi=\tau_{0} / 2$, the electron reaches its maximum energy $E_{\max }=0.255 a_{0}^{2} \mathrm{MeV}$. When $\xi>\tau_{0} / 2$, the electron is decelerated. One can also derive the time $t=$ $\frac{a_{0}^{2}}{4}\left[\xi-\frac{\tau_{0}}{2 \pi} \sin \left(\frac{2 \pi \xi}{\tau_{0}}\right)\right]+\xi$ and the distance $x=\frac{a_{0}^{2}}{4}\left[\xi-\frac{\tau_{0}}{2 \pi} \times\right.$ $\left.\sin \left(\frac{2 \pi \xi}{\tau_{0}}\right)\right]$ experienced by the electron from Eq. (2) when it arrives at $\xi$. Taking $\xi=\tau_{0} / 2$, one obtains the acceleration time and distance:

$$
t_{\mathrm{acc}}=\frac{\tau_{0}}{2}\left(1+\frac{a_{0}^{2}}{4}\right), \quad l_{\mathrm{acc}}=\frac{\tau_{0} a_{0}^{2}}{8} .
$$

Therefore, $l_{\text {acc }}$ is proportional to the laser intensity and it can be much larger than the laser duration when $a_{0}^{2} \gg 1$. For example, when the laser duration is $4 T_{0}$ and the intensity is $10^{22} \mathrm{~W} \mathrm{~cm}^{-2}, \quad l_{\text {acc }}=1.8 \mathrm{~mm}$, and $E_{\max }=$ $0.93 \mathrm{GeV}$. When the intensity is $10^{25} \mathrm{~W} \mathrm{~cm}^{-2}, l_{\text {acc }}=$ $1.8 \mathrm{~m}$, and $E_{\max }=0.93 \mathrm{TeV}$. For such a long acceleration distance, it is necessary to guide the laser to avoid the transverse spreading in space. 

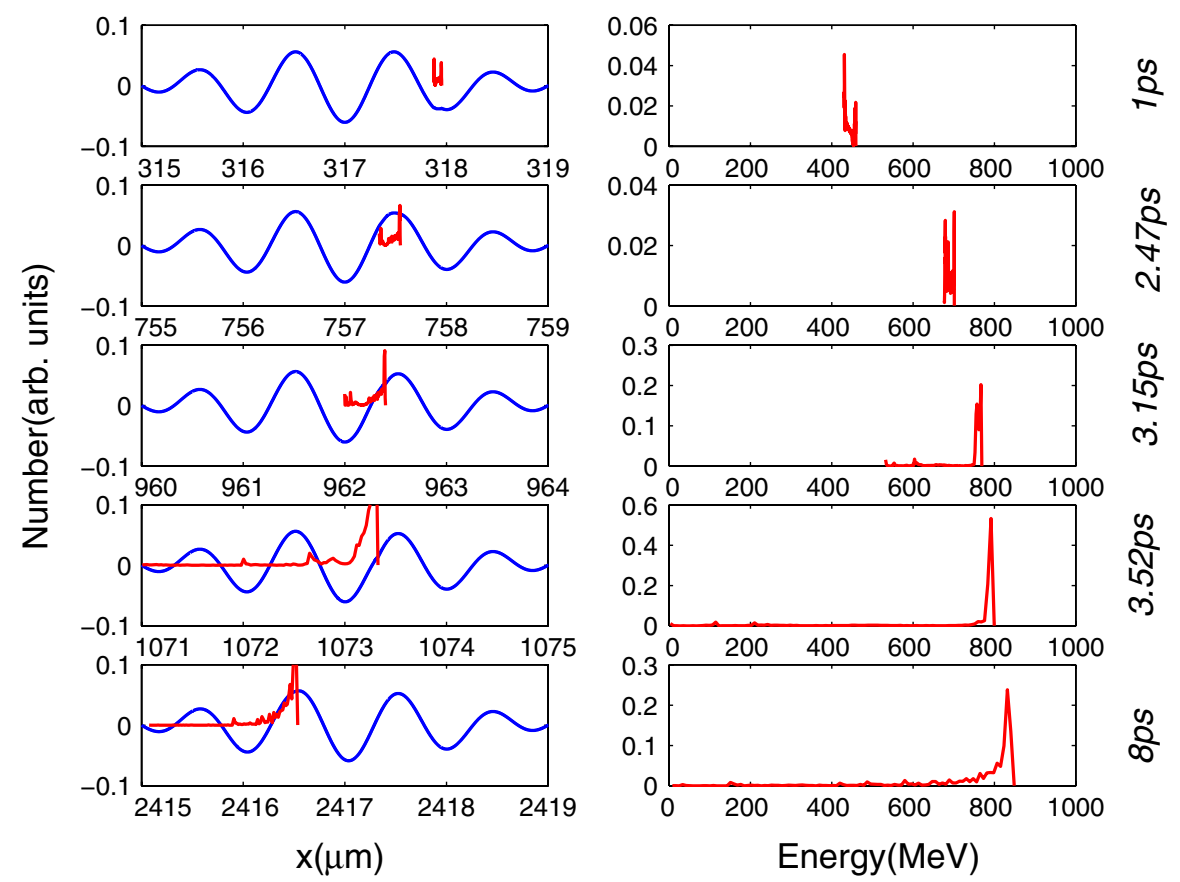

FIG. 2. (Color) Snapshots of the distributions of the electron number versus electron positions and energy are shown by the red curves. The blue curves in every plot of the left column are 1/1000 of the laser electric field $E_{y}$ normalized by $m_{e} c \omega_{0} / e$ to show the position of the electron beam in the acceleration or deceleration phase of the ponderomotive field, where the acceleration phase is within the leading edge of the laser pulse $\left(2 T_{0} / \mu \mathrm{m}\right.$ long $)$ and the deceleration phase is within the trailing edge of the laser pulse $\left(2 T_{0} / \mu \mathrm{m}\right.$ long). The four rows are the results at the time of $1,2.47,3.15$, and $8 \mathrm{ps}$, respectively.

\section{1D PIC SIMULATION RESULTS}

In the PIC simulations, we take the laser intensity of $10^{22} \mathrm{~W} \mathrm{~cm}^{-2}$ only to demonstrate the generation of about $\mathrm{GeV}$ electron beams. The simulation of electron beam generation of higher energy such as $\mathrm{TeV}$ demands a much longer computing time since $t_{\mathrm{acc}}$ is proportional to the laser intensity. In our 1D PIC simulations, the CP laser propagates along $+x$ direction with the wavelength $1 \mu \mathrm{m}$, the amplitude $a_{0}=60.4$, the duration $\tau_{0}=4 T_{0}$, and the waveform following Eq. (1). Thin source foils, with the plasma electron density $100 n_{c}$ and the thickness of $1-$ $100 \mathrm{~nm}$, are located at $x=20 \mu \mathrm{m}$. The laser wave front reaches the source foil at $3.3 \mathrm{fs}$ and the simulation time is 8 ps. The laser-blocking foil is not used in the 1D simulations.

The results for the $1 \mathrm{~nm}$ thick source foil are shown in Figs. 2 and 3. Figure 2 is the distribution of the electron number with electron positions and energy. Figure 3 is the distribution of electron energy with $\xi$. From Fig. 2 one can see that an electron bunch is trapped by the ponderomotive field. The acceleration time and distance are $3.15 \mathrm{ps}$ (see Fig. 3) and $0.945 \mathrm{~mm}$, respectively. Here, we take the acceleration time when any electrons enter the deceleration phase $(2<\xi<4)$ of the ponderomotive field. At $3.15 \mathrm{ps}$ its average energy, energy spread, and bunch width are about $800 \mathrm{MeV}, 0.24 \%$, and $0.4 \mu \mathrm{m}$, respectively. All of the electrons leave the acceleration phase of the pondero- motive field at $5.32 \mathrm{ps,}$ as observed in Fig. 3. This value is smaller than $t_{\mathrm{acc}}=6.09 \mathrm{ps}$ predicted by Eq. (3). The maximum energy observed at $5.32 \mathrm{ps}$ is $900 \mathrm{MeV}$, which is lower than the predicted value of $E_{\max }=930 \mathrm{MeV}$ by Eq. (2). The deviation of the single electron model from the

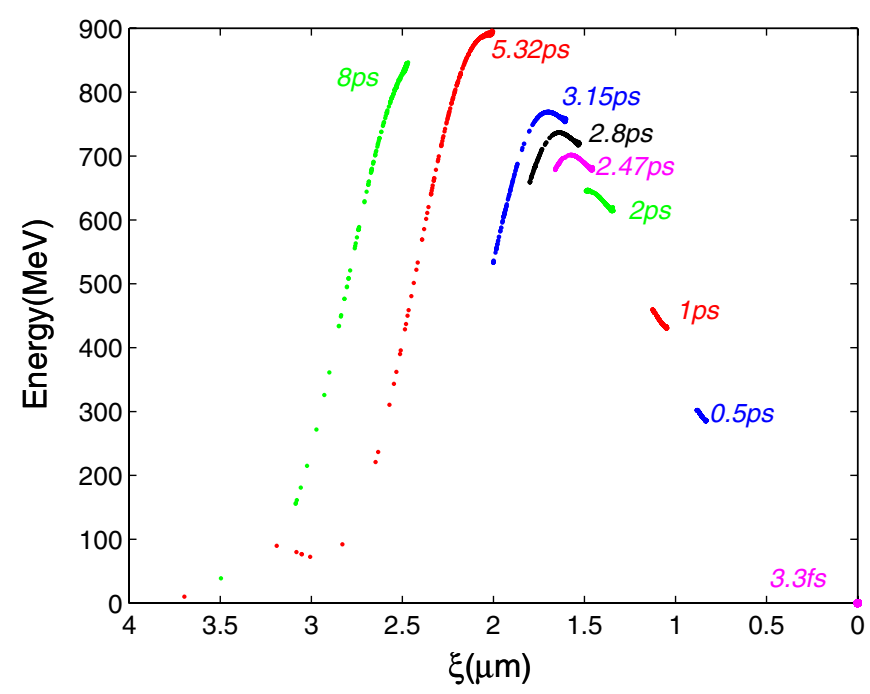

FIG. 3. (Color) Distributions of electron energy versus $\xi=t-$ $x$ at different time, where the acceleration phase of the ponderomotive field is within $0<\xi<2$ and the deceleration phase within $2<\xi<4$. 

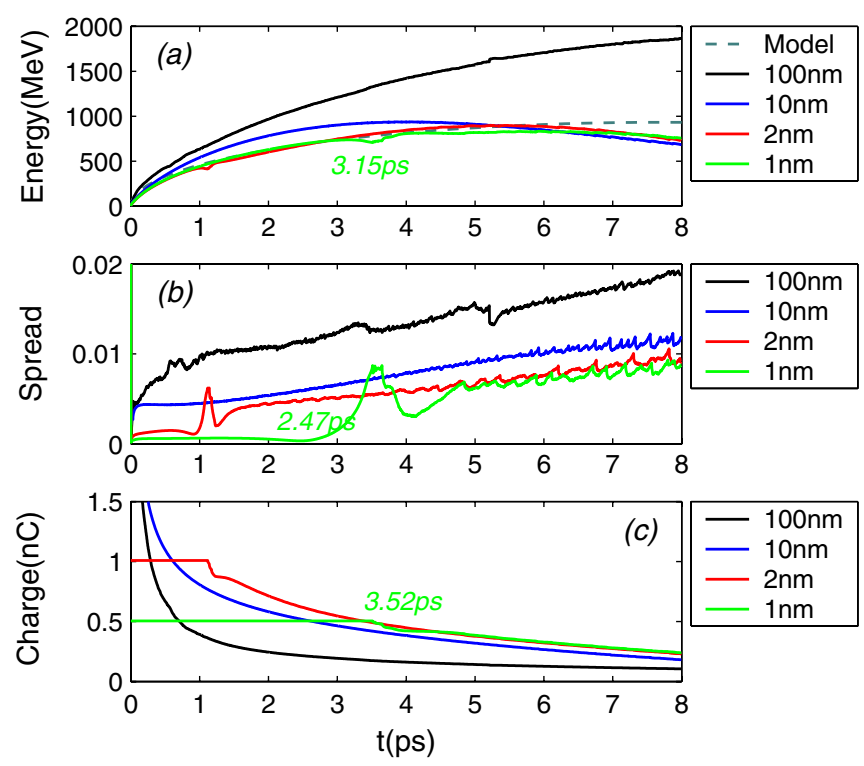

FIG. 4. (Color) The average energy, energy spread (the calculation method following Refs. [14,15]), and charges of the electron beam as a function of time, where the curves with different colors in every plot denote the source foils with different thickness, the broken line in (a) given by the single electron model, and only the electrons within the laser pulse $(0 \leq \xi \leq 4)$ are counted.

PIC results is due to the ignorance of the ES field in the model.

Figure 4 shows the temporal evolution of parameters of the trapped electron beam, where the energy spread is defined as $\Delta E /\langle E\rangle,\langle E\rangle$ is the average energy of the beam electrons, and $\Delta E$ is the square root of variance of the electron energy $[14,15]$. One can see from Fig. 4(a) that the electron beam energy with the $1 \mathrm{~nm}$ source foil is increased continuously with time until its maximum at about $5.32 \mathrm{ps}$, because all of the beam electrons leave the acceleration phase of the ponderomotive field at $5.32 \mathrm{ps}$, as seen in Fig. 3. Figure 4(b) displays that the energy spread goes down first until to the minimum at $2.47 \mathrm{ps}$ and then it grows on the whole. The reason is as follows. Because the electrons are injected from the front of the laser, the electrons initially located at the relative back positions in the foil (with smaller $x$ or larger $\xi$ ) meet the laser front first and are accelerated at the earlier time. They have larger velocities, as seen from the cases before $2.47 \mathrm{ps}$ in Fig. 3. Therefore, they can reduce the distance between them to the electrons at the relative front positions and consequently the energy difference is decreased, during the early accelerating process before $2.47 \mathrm{ps}$. Besides, the electrons at the rear tend to experience the larger ES field since the source foil ions are left behind the electron beam. This causes their velocities at $2.47 \mathrm{ps}$ to be exceeded by those at the front, as observed from the cases at $2.47 \mathrm{ps}$ in Fig. 3. At $2.47 \mathrm{ps}$ the energy spread reaches the minimum. At the later time the rear electrons begin to be decelerated (see the cases at and after $2.8 \mathrm{ps}$ in Fig. 3) and thus the spread rises. One can also observe in Fig. 4(b) that there is a jump in the spread at around $3.52 \mathrm{ps}$, when some electrons leave the laser pulse and the number of the electrons counted is decreased [see Figs. 2 and 4(c)].

The temporal evolution of parameters of the electron beams from the source foils with the thicknesses of 2, 10, and $100 \mathrm{~nm}$ is also plotted in Fig. 4. Figure 4(a) shows that the energy curves deviate from the single electron model more and more with the increase in foil thickness. Especially, when the thickness is $100 \mathrm{~nm}$, the electron beam energy is much higher than the model prediction. On one hand, the ES field is larger for the thicker source foils, which causes the simulation results to deviate from the single electron model results further. On the other hand, electrons absorb the laser energy at the laser leading edge and release energy at the trailing edge, which can change the laser pulse shape. When the source foil is thicker and the electron number is larger, this shape change is more obvious, as observed in Fig. 5. As a result, the acceleration time and gradient of the ponderomotive field increases further. This explains that the electron beam energy for the $100 \mathrm{~nm}$ source foil is rather higher than the model prediction.

In addition, it is observed from Figs. 4(b) and 4(c) that, with the increase in the source foil thickness, the number of trapped electrons drops and the energy spread rises. The reasons are as follows. First, the ponderomotive field should be larger than the ES field for the electron acceleration. Because of the larger ES field formed from the charge separation of the thicker source foil, the electron beam begins to be accelerated at the later time and it enters the deceleration phase of the ponderomotive field at the earlier time. Therefore, the trapped charge is smaller and the spread is larger at the same moment. Second, for the thicker source foil the size of the trapped electron beam

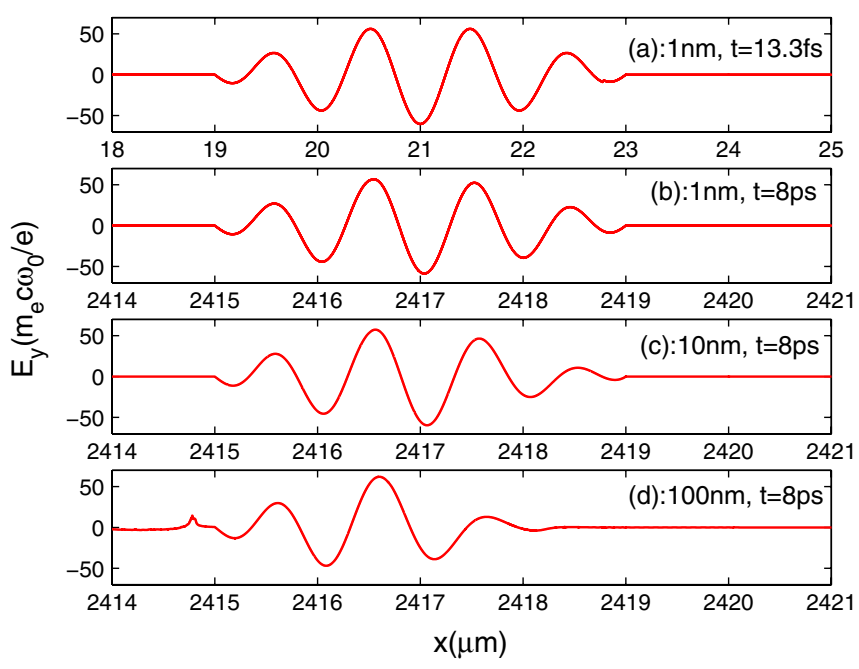

FIG. 5. (Color) Snapshots of the spatial distributions of the laser electric field interacting with source foils of different thickness. 
becomes larger and the electrons incline to experience different ponderomotive fields, which also causes larger energy spread.

The above results show that one can obtain high energy electron beams even with relatively thick source foils when the ponderomotive force can overtake the ES force, i.e. $a_{0}^{2} / \tau_{0}>8 \pi n_{e 0} D$. However, the thinner source foil with $a_{0}^{2} / \tau_{0} \gg 8 \pi n_{e 0} D$ affects the laser little. Therefore, its LPFA has better predictability and less complexity. Furthermore, the electron beams with the higher charge and quality can be produced from thinner source foils. It should be pointed that for LPFA another condition should be satisfied that $a_{0}>2 \pi n_{e 0} D$ [20-22] in order to drop the foil ions behind the electrons and the laser. This suggests that the laser intensity in LPFA should exceed that in the radiation pressure acceleration [23] or the phase stable acceleration [21].

\section{2D PIC SIMULATION RESULTS}

In the following, we present 2D PIC simulation results to demonstrate the scheme further. To increase the simulation cell size, we take the source foil with a thickness of $20 \mathrm{~nm}$ and a density of $5 n_{c}$, which is equivalent to the foil with thickness $1 \mathrm{~nm}$ and density $100 n_{c}$. The foil has a size of $6 \mu \mathrm{m}$ in the $y$ direction. To reduce the computing time, the laser duration $\tau_{0}$ is decreased to $2 T_{0}$ since $l_{\text {acc }} \propto \tau_{0}$, while keeping $a_{0}=60.4$ as before. The transverse distribution of the laser follows $\exp \left(-y^{2} / r_{0}^{2}\right)$ with $r_{0}=10 \mu \mathrm{m}$. The laser-blocking foil with the density $200 n_{c}$, thickness $100 \mathrm{~nm}$, and infinite transverse size is located at $x=$ $618.5 \mu \mathrm{m}$. It begins to experience the laser wave front at 1.998 ps. Figure 6 shows that an electron bunch is trapped and accelerated continuously. The electron beam still lies within the acceleration phase of the ponderomotive field at $2 \mathrm{ps}$. At $2.01 \mathrm{ps}$ it has an average energy of about $600 \mathrm{MeV}$, a spread of $0.7 \%$, and a bunch width of $0.4 \mu \mathrm{m}$, when separated from the laser. The density and energy distributions of the electron beam have little changes before (at 2 ps) and after (at $2.01 \mathrm{ps)} \mathrm{it} \mathrm{passes} \mathrm{through} \mathrm{the} \mathrm{laser-}$ blocking foil. The stable trapping and acceleration of the electron beam for such a long time (2 ps) and distance $(0.6 \mathrm{~mm})$ can be attributed to the relativistic effect. The electrons in LPFA are accelerated quickly to high energy and have large relativistic masses (e.g. the mass is $1175 m_{e}$ at $600 \mathrm{MeV}$ ), which overcomes the self-action between

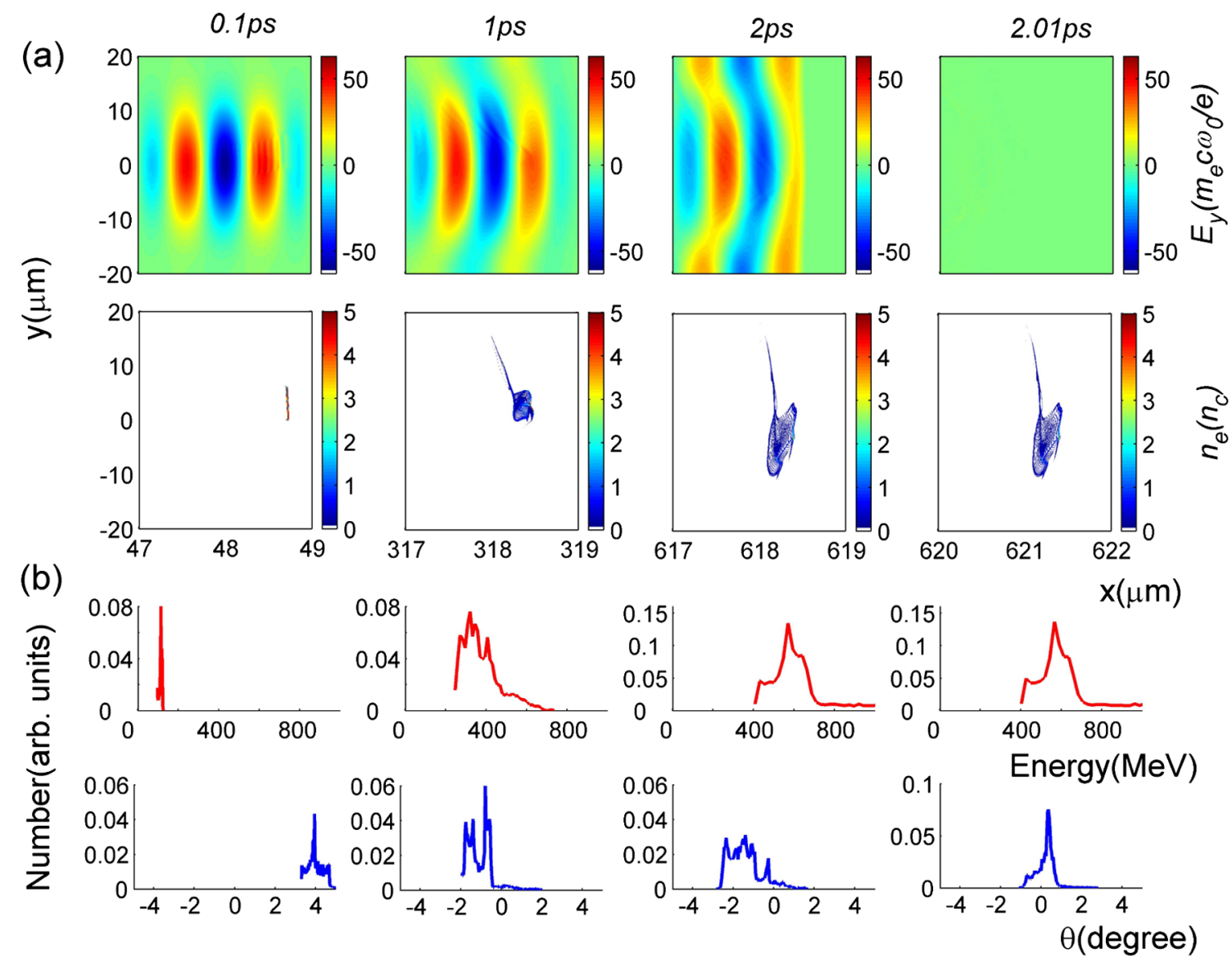

FIG. 6. (Color) (a) Spatial distributions of the laser electric field $E_{y}$ and electron density $n_{e}$, as well as (b) the distributions of the electron number as functions of electron energy and divergence angle at different time, where the laser-blocking foil is located at $x=618.5 \mu \mathrm{m}$. 
electrons and transverse instability [24,25] significantly. The transverse motion of the electron beam as a whole is also seen in Fig. 6. The average divergence angle changes with time, e.g., about $4^{\circ}$ at $0.1 \mathrm{ps}$, about $-1^{\circ}$ at $1 \mathrm{ps}$, and about $0^{\circ}$ at $2.01 \mathrm{ps}$, which can be explained as follows. The ultraintense laser field and ponderomotive field, in this case, dominate the self-action between the beam electrons. The transverse momentum or divergence angle of the electron beam is mainly determined by the laser field phase, which the beam is experiencing. As the beam experiences the different laser field phase, the evolution of the divergence angle with time appears. Especially, at $2.01 \mathrm{ps}$ the electron beam has just left the laser pulse and its transverse momenta become zero $\left(p_{y}=A_{y}=0\right.$ and $p_{z}=A_{z}=0$; the transverse ponderomotive force is much smaller than the longitudinal one for the laser parameters used here). Therefore, its average divergence angle is zero.

In the simulation above, a strong sheath electric field is seen at the back of the laser-blocking foil, when the laser interacts with it. However, this sheath field impacts the electron beam quite little, which can be seen by comparing the energy distribution at $2 \mathrm{ps}$ and at $2.01 \mathrm{ps}$ in Fig. 6. Because the electron beam with a speed near $c$ passes through the blocking foil quickly and this sheath field is local, the period of interaction of the electron beam with the blocking foil and the sheath field is quite short. Therefore, the electron beam energy nearly does not change. To make sure that the beam is affected by the blocking foil as little as possible while the laser is reflected completely, one should choose the blocking foil with a proper thickness. The optimum thickness can be taken as the critical transparent thickness with which the blocking foil can just reflect the laser pulse completely. The detailed discussion on the critical transparent thickness can be found in Refs. [21,22].

Next we preform two simulations to discuss the prepulse effect and the effect of the transverse size of the source foil, respectively. In the two simulations, we take different source foils and keep the same laser-blocking foil as used in Fig. 6. In simulation (a), we use a source foil with the infinite transverse size and the same other parameters as the source foil used in Fig. 6, to discuss the case of the source foil with the transverse size larger than the laser spot size. In simulation (b), we use a source foil with the thickness of $1 \mu \mathrm{m}$ (the length of the acceleration phase of the ponderomotive field or the leading edge of the laser pulse is $1 \mu \mathrm{m}$ ), the density of $0.1 n_{c}$, and the same other parameters as the source foil used in Fig. 6, to preliminarily estimate the prepulse effect on the foil expansion. It is assumed that this source foil is formed from the $1 \mathrm{~nm}$ thick foil with the density $100 n_{c}$ by its expansion. The distributions of the electron number with electron energy and divergence angle at 2.01 ps (just after the electron beam passes through the blocking foil) are shown in Fig. 7. One
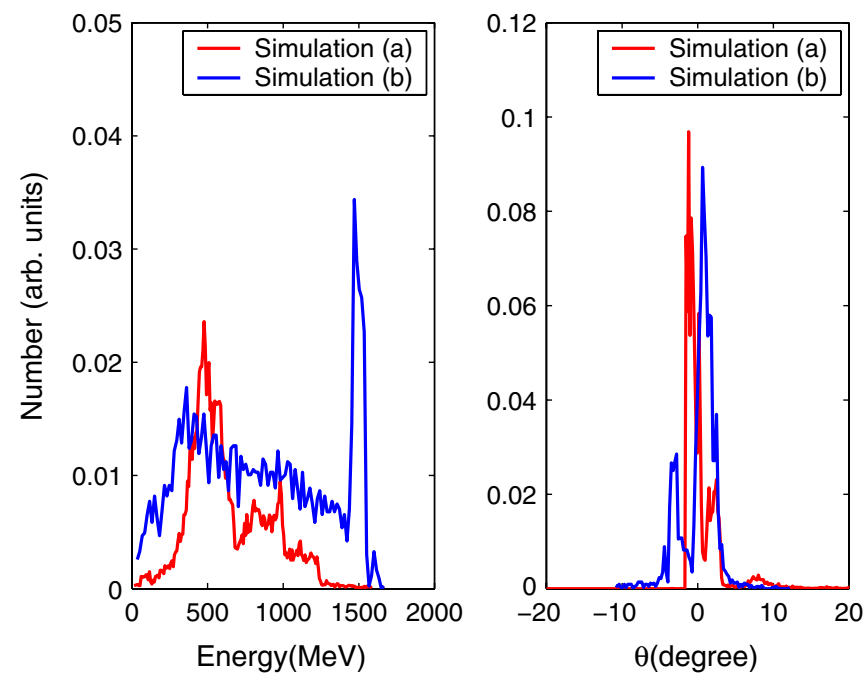

FIG. 7. (Color) Distributions of the electron number with electron energy and divergence angle at $2.01 \mathrm{ps}$ obtained from two respective simulations. In simulation (a), the source foil with the infinite transverse size is taken. In simulation (b), the source foil with the thickness of $1 \mu \mathrm{m}$ and the density of $0.1 n_{c}$ is taken.

can see that, compared with the results in Fig. 6, both the energy spread and the divergence grows in the two cases due to the increased volume (or area in $2 \mathrm{D}$ case) of the electrons injected into the ponderomotive field. In simulation (a), the increase in the energy spread and the divergence is not large and the average energy is decreased by about $100 \mathrm{MeV}$, compared with the results in Fig. 6. This indicates the source foil with a large transverse size can be used for LPFA. However, to achieve higher quality electron beams it is necessary to take a source foil with the transverse size smaller than the laser spot size. The contrast ratio of the laser may be required very high according to the result of simulation (b). We cannot estimate the requirement of the contrast strictly, because it depends on many factors such as the foil material, the prepulse intensity, the temporal gap between the prepulse and the main pulse (this factor may quite important), and so on. However, the requirement of the contrast should be somewhat higher than that in the radiation pressure acceleration [23] or the phase stable acceleration [21], since a thinner source foil is used in LPFA.

Besides, it should be pointed out that LPFA can also be applied to the positron acceleration since the effect of the lase ponderomotive force on positrons is the same with electrons.

\section{SUMMARY}

In conclusion, by the theory and the simulations we demonstrated that high energy ultrashort monoenergetic electron beams are generated efficiently by LPFA. The particle-in-cell simulations show that $\mathrm{GeV}$ electron beams are produced by the $10^{22} \mathrm{~W} \mathrm{~cm}^{-2}$ laser. It is predicted that 
$\mathrm{TeV}$ electron beams can be produced by the $10^{25} \mathrm{~W} \mathrm{~cm}^{-2}$ laser. The key in LPFA is that the acceleration distance in the ultraintense laser regime is large enough to allow one to separate the electron beam from the laser easily before the electron beam is decelerated. By use of the thin source foils, the electrons are trapped easily and the energy spread is reduced. In LPFA the electrons are accelerated to high energy quickly and have large relativistic masses, which overcomes their self-action significantly and keeps the acceleration stable for a long time. For the ultrashort ultraintense lasers discussed in this paper, LPFA has the larger acceleration field and may produce higher energy electron beams than LWFA.

\section{ACKNOWLEDGMENTS}

This work is supported in part by the National Natural Science Foundation of China (Grants No. 10734130, No. 10925421, No. 10935002, and No. 10974249), the National High-Tech ICF Committee in China, the National Basic Research Program of China (Grants No. 2007CB310406 and No. 2007CB815101), and CORE (Center for Optical Research and Education) at Utsunomiya University, Japan.

[1] C. DesRosiers, V. Moskvin, A. F. Bielajew, and L. Papiez, Phys. Med. Biol. 45, 1781 (2000).

[2] Y. Glinec J. Faure, V. Malka, T. Fuchs, H. Szymanowski, and U. Oelfke, Med. Phys. 33, 155 (2006).

[3] Y. Glinec, J. Faure, L. Le Dain, S. Darbon, T. Hosokai, J. J. Santos, E. Lefebvre, J. P. Rousseau, F. Burgy, B. Mercier, and V. Malka, Phys. Rev. Lett. 94, 025003 (2005).

[4] W. P. Leemans, C. G. R. Geddes, J. Faure, Cs. Toth, J. van Tilborg, C. B. Schroeder, E. Esarey, G. Fubiani, D. Auerbach, B. Marcelis, M. A. Carnahan, R. A. Kaindl, J. Byrd, and M.C. Martin, Phys. Rev. Lett. 91, 074802 (2003).

[5] Y. Shen, T. Watanabe, D. A. Arena, C.-C. Kao, J. B. Murphy, T. Y. Tsang, X. J. Wang, and G. L. Carr, Phys. Rev. Lett. 99, 043901 (2007).

[6] T. Tajima and J. M. Dawson, Phys. Rev. Lett. 43, 267 (1979).

[7] A. Pukhov and J. Meyer-ter-vehn, Appl. Phys. B 74, 355 (2002).
[8] S. P.D. Mangles, C.D. Murphy, Z. Najmudin, A. G. R. Thomas, J. L. Collier, A.E. Dangor, E. J. Divall, P.S. Foster, J. G. Gallacher, C. J. Hooker, D. A. Jaroszynski, A. J. Langley, W. B. Mori, P. A. Norreys, F. S. Tsung, R. Viskup, B.R. Walton, and K. Krushelnick, Nature (London) 431, 535 (2004).

[9] C. Geddes, C. Toth, J. van Tilborg, E. Esarey, C. Schroeder, D. Bruhwiler, C. Nieter, J. Cary, and W. Leemans, Nature (London) 431, 538 (2004).

[10] J. Faure, Y. Glinec, A. Pukhov, S. Kiselev, S. Gordienko, E. Lefebvre, J. Rousseau, F. Burgy, and V. Malka, Nature (London) 431, 541 (2004).

[11] W. Lu, C. Huang, M. Zhou, W. B. Mori, and T. Katsouleas, Phys. Rev. Lett. 96, 165002 (2006).

[12] W. Lu, M. Tzoufras, C. Joshi, F. S. Tsung, W. B. Mori, J. Vieira, R. A. Fonseca, and L. O. Silva, Phys. Rev. ST Accel. Beams 10, 061301 (2007).

[13] J. Faure, C. Rechatin, A. Norlin, A. Lifschitz, Y. Glinec, and V. Malka, Nature (London) 444, 737 (2006).

[14] W.-M. Wang, Z.-M. Sheng, and J. Zhang, Appl. Phys. Lett. 93, 201502 (2008).

[15] W.-M. Wang and Z.-M. Sheng, Phys. Plasmas 15, 013101 (2008).

[16] W.-M. Wang, Z.-M. Sheng, and J. Zhang, Laser Part. Beams 27, 3 (2009).

[17] V. I. Geyko, I. Y. Dodin, N. J. Fisch, and G. M. Fraiman, Phys. Plasmas 17, 023105 (2010).

[18] J. Meyer-ter-Vehn, A. Pukhov, and Z.-M. Sheng, in Atoms, Solids, and Plasmas in Super-Intense Laser Fields, edited by D. Batani et al. (Kluwer Academic/Plenum Publishers, New York, 2001), pp. 167-192.

[19] Wei Yu, V. Bychenkov, Y. Sentoku, M. Y. Yu, Z. M. Sheng, and K. Mima, Phys. Rev. Lett. 85, 570 (2000).

[20] X. Zhang, B. Shen, X. Li, Z. Jin, F. Wang, and M. Wen, Phys. Plasmas 14, 123108 (2007).

[21] X. Q. Yan, C. Lin, Z. M. Sheng, Z. Y. Guo, B. C. Liu, Y. R. Lu, J.X. Fang, and J.E. Chen, Phys. Rev. Lett. 100, 135003 (2008).

[22] A. A. Gonoskov, A. V. Korzhimanov, V. I. Eremin, A. V. Kim, and A. M. Sergeev, Phys. Rev. Lett. 102, 184801 (2009).

[23] A. P. L. Robinson, M. Zepf, S. Kar, R. G. Evans, and C. Bellei, New J. Phys. 10, 013021 (2008).

[24] M. Chen, A. Pukhov, Z. M. Sheng, and X. Q. Yan, Phys. Plasmas 15, 113103 (2008).

[25] M. Chen, A. Pukhov, T. P. Yu, and Z. M. Sheng, Phys. Rev. Lett. 103, 024801 (2009). 